\title{
Executive summary: advances in fetal and neonatal imaging
}

\author{
Dorothy Bulas
}

Received: 29 August 2011 / Accepted: 31 August 2011

(C) Springer-Verlag 2011

The Society for Pediatric Radiology hosted a gathering of experts in fetology and neonatology on Jan. 28-Jan. 30, 2011. The goal was to review current information on stateof-the-art fetal and neonatal imaging and its impact on perinatal care. Advances in research were discussed and future endeavors described. Challenges in perinatal and neonatal care were debated in open forums that included not only radiologists but neonatologists, maternal fetal specialists, pediatric surgeons, neurologists and genetic counselors. This conference helped demonstrate that pediatric radiologists are an important collaborator in the evaluation and advanced care of the fetus and neonate.

The evaluation and care of the fetus and neonate have exploded during the last 2 decades. Advances in prenatal testing, high-resolution US, rapid-sequencing MRI and even 3-D CT for skeletal assessment have provided exquisite detail regarding the fetus and intrauterine environment. The ability to assess the health of the fetus and identify anomalies has changed the practice of both obstetrics and neonatology. By identifying the fetus with anomalies or at risk for intrauterine compromise, management can be guided by appropriate specialists with resultant improved outcome. Advances in US, CT and MRI have also dramatically improved the evaluation of the neonate noninvasively, allowing for the successful evolution of innovative neonatal therapies.

The 20th century will be known as the century of the human genome. Prenatal testing continues to rapidly evolve and helps direct the need to further image the fetus. The earlier diagnostic testing is initiated, the higher the rate of

D. Bulas $(\bowtie)$

Children's National Medical Center,

111 Michigan Ave. NW,

Washington, DC 20010, USA

e-mail: dbulas@childrensnational.org aneuploidies compared to delivery caused by spontaneous losses throughout pregnancy. Screening tests provide a risk estimate without the risk of pregnancy loss, while diagnostic tests provide a definitive answer but with the potential risk of pregnancy loss by chorionic villus sampling (CVS) or amniocentesis. The goal of integrated and sequential firsttrimester nuchal translucency and second-trimester triple test or quad test is to provide a relatively high sensitivity and specificity screening test. Specificity with these tests, however, remains relatively low, requiring invasive testing or imaging to identify true positives. Invasive diagnostic methods include (1) amniocentesis with its low risk of miscarriage, technically uncomplicated but performed in the second trimester, (2) CVS with its low miscarriage risk, technically more complicated, and (3) early amniocentesis with the highest risk of miscarriage, with fetal morbidity and technical concerns. Free fetal DNA (ffDNA) in maternal circulation might become a way to test for selected conditions noninvasively in the future.

$U S$ is the primary method for imaging fetal anatomy and is used throughout pregnancy to establish fetal number, gestational age and placental health and to address clinical concerns for aneuploidy, poor fetal growth and abnormal morphology.

Higher-resolution transducers, transvaginal imaging and advances in 3-D volumetric technology have improved the reliability of US to identify complex anomalies as early as the first trimester. US is particularly useful in the evaluation of fetal kidneys, extremities and face. Doppler evaluation allows for assessment of fetal health including intrauterine growth retardation.

Advances in fetal echocardiography including Doppler and 3-D/4-D images have provided further understanding of the hemodynamics of the fetus and pathophysiology of the fetal heart. Dedicated echocardiography should be considered when an extracardiac malformation is identified; 
family history of congenital heart defects, particularly in a parent or sibling, are present; a genetic disease associated with heart defects is suspected; or a maternal disease associated with fetal heart defects such as diabetes or lupus is present. High-resolution studies can now be successfully performed even in the first trimester transvaginally. Early diagnosis of cardiac anomalies in combination with other extracardiac abnormalities can improve counseling concerning long-term outcome. Understanding the natural history of cardiac lesions has led to the proposal of in utero interventions for certain cardiac defects. Specifically, in some cases of hypoplastic left heart syndrome, dilatation of the aortic valve prenatally can result in improved biventricular postnatal outcome with the left ventricle supporting systemic circulation.

Factors such as fetal lie, overlying bone, maternal obesity, and oligohydramnios can interfere with the sonographic evaluation of the fetus. $M R I$ is an alternative modality to evaluate the fetus as it uses no ionizing radiation and has excellent tissue contrast, large field of view and multiplanar capabilities. The development of faster scanning techniques allows studies to be performed without sedation in the second and third trimesters with superior contrast in multiple planes.

MRI is used as a complementary study only when US results are inadequate for diagnosis or when MR imaging is expected to provide important additional information. With advances in the perinatal treatment of fetal abnormalities there is an increasing need for precise depiction of these abnormalities. Fetal MRI not only confirms the presence of lesions noted by US but can demonstrate additional subtle anomalies. Areas in which MRI is particularly helpful include evaluation of the fetal brain (anomalies, infarct, hemorrhage), chest (CPAM, sequestration, diaphragmatic hernia), craniofacial area (central clefts) and airway (teratoma, lymphangioma). Evolving applications include the assessment of GI/abdominal wall anomalies, GU abnormalities, particularly in the presence of oligohydramnios, and musculoskeletal lesions. MR images can provide reproducible measurements of lung volumes and airway compression that can aid in assessing risk outcomes. Experts including pediatric surgeons, neurologists, neurosurgeons, ENT specialists and neonatologists who are uncomfortable interpreting sonograms can review MR imaging and provide additional expertise in the care of the fetus. This information helps appropriate specialists counsel families when difficult decisions must be made with regard to early and potentially invasive intervention and allows for optimal delivery planning and postnatal procedures. EXIT to airway procedures for neck masses, in utero surgery for meningomyelocele repair or the resection of vascular masses have succeeded because of the combined work of specialists who have access to the multiplanar images MR provides of the fetus.
In the future, exciting advances in MR techniques including quantitative measurements, diffusion-weighted imaging, spectroscopy and functional imaging might provide specific information that will improve the detection and monitoring of the fetus at risk.

Once the fetus is born, imaging advances in fluoroscopy, US, MRI and CT continue to provide improved care of the neonate. Fluoroscopy can quickly identify life-threatening abnormalities of the GI tract such as malrotation, meconium ileus and atresias. US remains a cornerstone in neonatal imaging of the brain, chest and abdomen because of the lack of radiation, the portability and the lack of sedation requirements. MRI-compatible incubators have improved the safety of performing the exam on unstable infants, with advances in sequences providing specific physiological, functional and quantitative information. Multidetector CT with multi-planar reconstruction and 3-D volume rendering, while less used in neonates because of the high radiation burden, can provide critical information quickly in the unstable infant with pulmonary or vascular ring abnormalities.

Brain injury in the preterm and term infant is a complex and heterogeneous process. We are learning that many cases of hypoxic ischemic encephalopathy (HIE) are related to events arising prior to onset of labor. Fetal heart rate monitoring, Doppler assessment, biophysical profile and umbilical arterial blood analysis can be used to assess the presence of intrapartum HIE. Advances in postnatal assessment of neonatal brain injury by US using linear transducers, Doppler and supplemental views can improve the sensitivity of identifying ischemic injury and are useful for the follow-up assessment in these unstable patients. However, MRI advances including MR-compatible incubators, MRA/ MRV, diffusion-weighted imaging, spectroscopy, diffusion tensor imaging and quantitative volumetric imaging have made MR the gold standard for the evaluation of neonatal brain injury. Advanced postnatal neuroimaging techniques provide the perinatologist and neonatologist with improved understanding of the timing and pathophysiology of perinatal brain injury, aiding in the development of therapeutic strategies such as hypothermia and neuroprotective agents that improve long-term sequelae.

Radiologists need to be familiar with the fetal and neonatal appearance of anomalies and abnormalities and also need to understand the long-term implications of these findings. Major advancements in imaging can result in improvement in the care of the fetus and infant, resulting in better-quality outcome and long-term survival.

Disclaimer The supplement this article is part of is not sponsored by the industry. Dr. Bulas has no financial interest, investigational or off-label uses to disclose. 